\title{
Materias primas y formas líticas del Auriñaciense Arcaico de la Cueva del Castillo (Puente Viesgo, Cantabria)
}

\author{
V. Cabrera Valdés* ${ }^{\star}$, M. Lloret Martínez de la Riva** \\ y F. BERNALDO dE QUIRÓs ***
}

\section{RESUMEN}

El yacimiento de la Cueva del Castillo, en las campañas de excavación que se vienen realizando desde 1980 ha proporcionado materiales líticos realizados en diversas materias primas para el nivel 18, considerado como Auriñaciense desde las primeras excavaciones organizadas por el I.P.H. El estudio que aquí presentamos está basado en la serie de útiles líticos y no sobre la totalidad de la industria con el fin de realizar una comparación de orden litológico en los diferentes grupos de útiles que aparecen en dicho nivel.

\section{ABSTRACT}

The archaelogical site of Castillo cave, in the new diggins that are in process since 1980, offers several raw materials for lithic industry in level 18 attributed to Aurignacien since the first excavations of I.P.H. We study in this work only the tipological tools and their raw materials, in order to make a lithological comparison among different tool groups.

\section{PALABRAS CLAVE}

El Castillo, Auriñaciense arcaico, industria lítica, materias primas, grupos tipológicos.

* Departamento Prehistoria e Historia Antigua, U.N.E.D., Madrid.

** Alumna de tercer ciclo del Departamento Prehistoria, U.N.E.D., Madrid.

*** Área de Prehistoria, Universidad de León, León. 


\section{INTRODUCCIÓN}

Los resultados que se presentan a continuación han sido posibles gracias a la financiación del proyecto de la DGICYT PB92-0562 que enmarca los trabajos sobre la transición del Paleolítico Medio/Superior en la Cornisa Cantábrica, proyecto que se realiza desde la U.N.E.D.

La secuencia de la Cueva del Castillo (Puente Viesgo, Santander) representa una de las más amplias conocidas en la Península lbérica, conteniendo una sucesión completa de ocupaciones paleolíticas que se subdividieron en 26 capas en las que alternaban niveles estériles con los de ocupación humana, llegando alcanzar una potencia estimada según Obermaier de 18-20 m. en las excavaciones del I.P.H. de 1910-1914.

En los años 80 , después de la revisión efectuada por V. Cabrera Valdés se determinaron los siguientes niveles culturales: dos niveles con Paleolítico Medio Antiguo, un nivel Achelense Superior, dos niveles Musterienses, dos niveles Auriñacienses, dos del Perigordiense Superior, uno del Solutrense Medio, tres de diferentes etapas del Magdaleniense y finalmente un Aziliense.

Las nuevas excavaciones dirigidas por $V$. Cabrera Valdés han sido realizadas cerca de la entrada de la cueva, en la proximidad del ángulo de los cortes transversal y longitudinal dejados por Obermaier (Cabrera Valdés et al., 1993; 1996). Éstas han permitido establecer una correlación entre esta parte del lugar y la secuencia estratigráfica de Obermaier. Se ha respetado la numeración de los niveles dada por Obermaier (Obermaier, 1925, Cabrera Valdés, 1984) aunque en ellos se hayan distinguido subniveles arqueológicos o sedimentarios.

Las nuevas excavaciones han aportado un espesor de 4 a $5 \mathrm{~m}$ entre los niveles 17 y 23 del corte transversal sobre una superficie total de $40 \mathrm{~m}^{2}$; teniendo en cuenta la presencia de numerosos bloques de desprendimiento hacia la entrada de la cueva, la superficie real de la ocupación en esta zona queda reducida a $24 \mathrm{~m}^{2}$. En este área se ha excavado en horizontal la capa 18 de la estratigrafía de Obermaier.

Las excavaciones también han aportado entre los niveles 13 y 26 del corte longitudinal, una superficie de $3 \mathrm{~m}^{2}$ y un espesor de alrededor de $6 \mathrm{~m}$. Esta zona más reducida ha proporcionado materiales de las capas 16 y especialmente de la capa 18 (ambas Auriñacienses).

Los niveles superiores de la secuencia no han podido ser excavados debido a que están muy degradados y cubiertos de desprendimientos. Un estudio geofísico ha establecido que aún quedan $7 \mathrm{~m}$ de sedimento debajo del nivel 23, antes de aparecer el substrato rocoso. 
El nivel 18 "auriñaciense arcaico» que nos ocupa aquí presenta una potencia variable por la deformación producida por grandes bloques. En las zonas menos alteradas presenta $20-30 \mathrm{~cm}$. Está compuesta por arcillas pardas ricas en materia orgánica englobando cantos y bloques de caliza más abundantes hacia la entrada de la cueva. Su base se caracteriza por una concentración importante de materia orgánica (carbón de madera) y una débil cantidad de elementos detríticos calcáreos. En la zona intermedia se encuentra un lecho arenoso calcáreo de 6 a $8 \mathrm{~cm}$. de espesor.

El estudio sedimentológico sugiere que los fenómenos de gelivación coetáneos de la formación de este nivel han sido moderados en la base y más intensos a techo, en todo caso no demasiado intensos, siempre de menor entidad que en el nivel 20.

Las características paleoclimáticas de este nivel corresponden a un clima poco frío en la base que pasa a condiciones de temperatura algo más rigurosas en el techo.

Respecto a la humedad, puede considerarse ésta dentro de un clima húmedo, algo más importante en una pulsación interior coincidente con los mayores aportes por escorrentía.

Al final de la sedimentación de este nivel se produce el mayor derrumbe de bloques del techo observable en la cueva quedando dicho nivel sellado por un gran desprendimiento posterior a la ocupación humana.

Durante las excavaciones de los años 80 , éste nivel fue dividido en tres subniveles según las bases sedimentológicas y según la importancia más o menos grande de restos antrópicos: 18 b1 a techo, 18 b2 y $18 \mathrm{c}$ en la base. Las sucesivas revisiones a las que hemos sometido los distintos materiales arqueológicos de la capa 18 , nos hacen considerar los subniveles 18 b1 y 18 b2 como uno mismo, denominado 18 b.

La industria que ha sido descubierta es tipológicamente homogénea en ambos subniveles y se caracteriza sobre todo por un porcentaje importante de raspadores, como la descubierta por Obermaier a principio de siglo.

La industria ósea está representada por numerosos elementos descubiertos en la base del subnivel (18c): el extremo distal de un «cincel» que presenta una serie rítmica de trazos horizontales ("marcas de caza"), cuyos prototipos se encuentran en niveles del auriñaciense antiguo europeo. El extremo distal de una azagaya en asta, así como fragmentos de asta alargados que se pueden relacionar con la fabricación de azagayas. Estos materiales también están presentes en la colección antigua, junto a una colección de 10 puntas de base hendida y sección losángica. 


\section{LAS MATERIAS PRIMAS DEL NIVEL 18 (Auriñaciense Arcaico):}

Para la identificación de los materiales se ha seguido un primer criterio macroscópico determinado por colores y la organización de éstos, la textura, la transperencia u opacidad de la misma, la presencia de corteza y su espesor así como las inclusiones en la propia materia de determinados elementos, que han ayudado junto con los remontajes líticos realizados en los restos de la talla a identificarlos y definirlos en tipos y subtipos. Este análisis ha sido aplicado también al nivel musteriense donde se ha observado que los tipos y subtipos de materia prima que aparecen en dicho nivel por el momento, puesto que aún se sigue excavando parte de este nivel, no presentan ninguna diferencia respecto al nivel auriñaciense que aquí se presenta. Asimismo la identificación de la materia prima como el origen de ésta se ha extendido a otros yacimientos próximos (cueva de La Pila en Cuchia, Cantabria) con la intención de precisar el radio de acción de los hombres prehistóricos en la recogida y selección de la materia prima no sólo en un determinado momento sino en momentos culturales próximos y distantes en el tiempo para ver los cambios que se producen en las formas de adquisición de una de las principales bases para la producción del utillaje.

Las materias primas encontradas e identificadas en el nivel $18 \mathrm{del}$ Auriñaciense arcaico de la cueva del Castillo se estudiaron conforme a una primera distinción en varias capas así; $17 \mathrm{~d}, 18$ base, 18 b1, 18 b2 y $18 \mathrm{c}$. No obstante, después de efectuadas diversas revisiones de los materiales arqueológicos el Auriñaciense arcaico ha sido considerado como dos subniveles: el subnivel 18b que englobaría a las capas $17 \mathrm{~d}, 18$ base, 18 b1 y 18 b2; y el subnivel 18c (Cabrera Valdés et al., 1996).

Cómo ambos subniveles no presentan ninguna diferencia en cuanto a la selección y formas de adquisición de la materia prima nos parece más acertado y más cómodo para el lector describir los tipos y subtipos de todo el nivel 18. Y pasar a examinar posteriormente el diferente tratamiento de las materias primas en la fabricación de determinados útiles del subnivel $18 \mathrm{~b}$ como del subnivel $18 \mathrm{c}$.

La industria lítica del Auriñaciense arcaico ha sido trabajada bajo unos nódulos que han sido escogidos para un determinado utillaje como iremos viendo en el desarrollo de los diferentes materiales que se presentan a continuación.

\section{LA CUARCITA}

Tres variedades de cuarcita aparecen en el nivel 18. La cuarcita de cantos pequeños y grano muy fino " $\mathrm{C} 1$ " y que encontramos en mayor 
cantidad, y la cuarcita verde de cantos mayores que denominaremos "C2" y la cuarcita gris opaca "C3".

La cuarcita "C1" que se encuentra en forma de canto pequeño de río $(5 \mathrm{cms}$.) tiene tres subtipos conforme al tipo de grano de su textura:

Subtipo «C1.1»

De grano muy fino, compacta y suave, presenta minúsculas puntuaciones de manganeso y una corteza lisa, suave y fina $(0,5 \mathrm{~mm})$ de color grisáceo y tendente al mismo color que el interior de la propia materia. De buena calidad para la talla. Se presenta en la siguiente gama de colores:

- Cuarcita roja con puntos negros.

- Cuarcita roja con puntos negros y manchas grises.

- Cuarcita roja hacia gris hacia blanca con líneas negras y puntos negros.

- Cuarcita gris hacia roja con puntos negros.

- Cuarcita gris verdoso con puntos negros.

Subtipo "C1.2»

De grano fino pero más tosco y menos compacto que el subtipo precedente. Presenta igualmente inclusiones de minúsculas puntuaciones de manganeso. La corteza es fina y del mismo color o tendente al color del interior de la materia. De buena calidad para la talla. Se ha observado que algunas piezas han sufrido alteración térmica.

Los colores que encontramos en este subtipo son:

- Cuarcita roja con puntos negros.

- Cuarcita gris verdosa con puntos negros.

- Cuarcita gris veteada en gris más oscuro con puntos negros.

- Cuarcita nieve sucio hacia naranja con puntos negros.

\section{Subtipo "C1.3"}

De textura de grano medio con minúsculas puntuaciones de carbonatos, de calidad mediocre y de color gris con puntos negros y puntos brillantes. 
La cuarcita "C2" se encuentra en forma de canto de río mediano hacia grande sobrepasando los $10 \mathrm{cms}$. Se reconoce fácilmente por su color verde opaco, su textura de grano fino y su homogeneidad presentando además pequeños granos de cuarzo. Su corteza es fina $(0,5 \mathrm{~mm})$, lisa, suave y tiene el mismo color que el interior de la materia. $Y$ aunque es de buena calidad para la talla, no obstante hemos distinguido dos subtipos según la homogeneidad de su textura que permite realizar la talla de forma más óptima:

Subtipo «C2.1»

Cuarcita de color verde opaco con gránulos pequeños negros y con algún punto brillante de grano fino y homogénea siendo más áspera que el subtipo "C2.2" que se presenta como una cuarcita de color verde opaco más claro, más fina y más homogénea que el anterior presentando una corteza del mismo color y tan fina que casi no se identifica el límite entre ésta y la propia materia.

Algunas piezas presentan una pátina muy homogénea de color verdoso y otras de color marrón que no ha de confundirse con ningún otro tipo, simplemente es una alteración de la propia materia así como aquellas piezas que presentan oxidación dando un aspecto herrumbroso.

La cuarcita "C3" es la tercera variedad que encontramos de esta materia aunque en escasa proporción. Se trata de una cuarcita margosa que se caracteriza por su grano fino muy compacto con inclusiones de minúsculas puntuaciones de manganeso y cuyo color es gris opaco. Es apta para la talla. Por el momento desconocemos su procedencia y la forma de presentarse. Hemos distinguido dos subtipos según presente la textura más o menos compacta:

Subtipo “C3.1"

Cuarcita muy compacta de color gris opaco con puntos negros, y cuarcita gris verdoso.

Subtipo "C3.2"

Cuarcita gris opaco, menos compacta que la anterior, con una franja rojiza fina con puntos negros. 


\section{LA CALIZA}

La caliza que aparece en el nivel 18 es una caliza negra muy compacta, suave, con corteza grisácea y fina. Se encuentra en forma de canto de tamaño mediano hacia grande y en una única variedad que denominamos «K1». Su calidad para la talla es buena tendente a mediocre. Debido a las diversas alteraciones que ha sufrido su aspecto, aparece en un color gris ceniza.

\section{EL SILEX}

El silex aunque en baja proporción está presente en el utillaje del nivel 18 en dos variedades. La primera variedad, y siguiendo la nomenclatura dada en anteriores trabajos (Lloret, 1995), es el silex tipo "S1" de textura de grano muy fino y homogéneo aunque debido a la variedad en su opacidad hace que lo clasifiquemos en dos subtipos:

\section{Subtipo «S1.1"}

De textura muy fina, homogénea y suave con inclusiones de minúsculas puntuaciones e hilillos de manganeso, presentándose en color gris y hueso:

- Silex gris claro con puntos negros e hilillos cortos en gris y blanco. Con algún punto brillante.

- Silex gris claro con puntos negros y algún punto brillante.

- Silex gris claro con manchas beiges y puntos grises.

- Silex gris con puntos negros y brillantes, y gránulos muy pequeños beiges.

- Silex gris con puntos negros y puntos brillantes.

- Silex gris con puntos negros, línea fina azulona, y mancha beige.

- Silex gris oscuro con puntos negros y grises difuminados.

- Silex hueso con manchas grises, puntos rojizos e hilillos cortos blancos.

- Silex hueso con manchas grises claras y puntos rojizos.

La corteza es porosa y fina $(0,5 \mathrm{~mm})$. Algunas piezas presentan neocorteza así como desilificación, oxidación y gelifracción. Se presenta en 
forma de pequeño canto de río y es de muy buena calidad para el trabajo de talla.

\section{Subtipo «S1.2»}

De textura muy fina, homogénea, suave y con inclusiones de minúsculas puntuaciones de manganeso como el anterior subtipo sin embargo, éste se caracteriza por una opacidad fuerte. Algunas piezas han sufrido desilificación e incluso encontramos piezas con una ligera pátina del mismo color de la propia materia, que por otra parte no ha impedido ser identificadas en este subtipo. Se presenta en la siguiente grama de colores:

- Silex marrón opaco vetado con línea marrón oscura y línea marrón clara. $Y$ con puntos negros.

- Silex gris opaco con líneas finas de color gris oscuro y con puntos negros.

- Silex gris oscuro con algún punto brillante.

- Silex beige opaco con manchas grises.

La corteza porosa y fina $(0,5 \mathrm{~mm})$ aunque también aparece lisa y fina $(0,5-1 \mathrm{~mm})$. Se presenta en cantos pequeños de río y es de buena calidad para la talla.

La segunda variedad de silex es el tipo «S3" ya definido en otros trabajos como un silex de calidad mediocre para el trabajo de talla, de textura de grano fino, homogéneo con minúsculas puntuaciones de manganeso y sílice. Se presenta generalmente desilificado y su corteza es porosa. Por el momento desconocemos su procedencia y la forma de presentarse. (Lloret, 1995; Cabrera Valdés et al., 1996). El color oscila entre un silex beige/hueso con algún punto pequeño rojo, un silex blanco con puntos negros y algún punto brillante, y un silex blanco con puntos negros difuminándose estos últimos.

\section{LA OFITA}

La ofita se presenta en calidades de grano fino y pequeños gránulos de piroxeno, con corteza fina $(0,5 \mathrm{~mm}-1 \mathrm{~mm})$ y de calidad buena para la talla la presentamos en tres subtipos según el aspecto de su color que varía de beige a gris: 
Subtipo "Of1", ofita beige con gránulos pequeños negros.

Subtipo "Of2", ofita gris verdosa con gránulos negros y gránulos beiges.

Subtipo "Of3", ofita beige con gránulos negros y blancos muy pequeños.

\section{LA LIMONITA}

Es la única variedad de mineral de hierro, "MFe1", que aparece en el nivel 18. Su aspecto es de color marrón, muy suave con corteza suave y fina, y cuya calidad para el trabajo de talla es buena tendente a mediocre.

\section{LA ARENISCA}

La arenisca de grano fino con inclusiones de puntos pequeños negros, de color beige y corteza fina ( $1 \mathrm{~mm}-1,5 \mathrm{~mm}$ ) aparece con una única variedad, «A1», en forma de canto.

Por último, la presencia anecdótica de un CUARZO blanco microcristalino, «Q1», que aparece en el subnivel $18 \mathrm{~b}$.

\section{EL UTILLAJE DEL NIVEL 18 (Auriñaciense arcaico)}

Nuestra intención es realizar una comparación de orden litológico entre los dos subniveles y comprobar si existen o no diferencias en la utilización de las materias primas para un determinado útil o grupos de útiles. Para tal efecto presentamos el utillaje según la materia prima de los subniveles.

Los grupos de útiles según materias primas del subnivel 18b:

De un total de 254 útiles tenemos que $187(73,62 \%)$ son fabricados en cuarcita; $28(11,02 \%)$ en silex; $28(11,02 \%)$ en caliza; $5(1,96 \%)$ en ofita; 5 $(1,96 \%)$ en limonita, y $1(0,39 \%)$ en cuarzo.

\section{Grupo de raspadores:}

47 han sido los raspadores fabricados en cuarcita siendo 43 de ellos del tipo «C1». En silex se han registrado 10 siendo 8 del tipo « $\mathbf{S} 1$ ", en caliza tenemos 18 raspadores, en ofita del tipo Of1 aparecen 2 útiles de este grupo, y 1 raspador fabricado sobre limonita. 


\section{Grupo de piezas retocadas:}

En este grupo englobamos tanto a las raederas como aquellas piezas que presentan retoque continuo.

Se han registrado 114 piezas en cuarcita en este grupo siendo 97 del tipo C1. En silex 17 son las piezas a destacar siendo del tipo «S1"11. En caliza tenemos 5 piezas, en ofita del tipo Of1 y en limonita 2 piezas respectivamente y en cuarzo una sola pieza.

Grupo de útiles compuestos:

En este grupo asociamos aquellas piezas retocadas que a su vez presentan una muesca, un raspador, una raedera $u$ otro útil.

Los útiles de este grupo son escasos así sólo se han registrado dos en cuarcita del tipo "C1», y otros dos en ofita del tipo «Of2» y en limonita.

\section{Grupo de piezas escotadas:}

Las muescas y muesquitas están presentes en cuarcita así tenemos 8 piezas siendo 5 de ellas del tipo "C 1 ", y una en limonita.

Grupo de piezas denticuladas:

En cuarcita del tipo "C1" tenemos cinco piezas y tres en caliza.

\section{Grupo de armaduras:}

Este grupo representado bajo un índice muy débil se caracteriza por una única pieza de borde rebajado en cuarcita del tipo "C1".

Grupo de piezas truncadas:

Una única pieza en caliza.

\section{Puntas:}

Como el grupo anterior sólo está presente con una punta de cuarcita del tipo «C1».

\section{Perforador y microperforador:}

Encontramos dos perforadores, uno en cuarcita del tipo "C1" y otro en silex del tipo "S1" así como un microperforador en cuarcita del tipo "C1".

\section{Buriles:}

Seis buriles en cuarcita del tipo "C1" teniendo que destacar que cinco son del subtipo «C1.2".

Los grupos de útiles según materias primas del subnivel 18c:

De un total de 216 útiles tenemos que $183(84,72 \%)$ son fabricados en cuarcita, $25(11,57 \%)$ en silex; $7(3,24 \%)$ en ofita, y $1(0,46 \%)$ en limonita. 


\section{Grupo de raspadores:}

Se han registrado 66 raspadores en cuarcita siendo 59 del tipo «C1». En silex tenemos 10 siendo 7 del tipo «S1». La proporción disminuye en la ofita donde sólo hay dos, uno del tipo "Of1" y otro del tipo "Of2", y un único raspador en limonita.

Grupo de piezas retocadas:

Como en el subnivel $18 \mathrm{~b}$ incluímos tanto a las raederas como aquellas piezas de retoque contínuo.

En cuarcita se han registrado 86 piezas siendo 69 del tipo "C1" y una del tipo "C2". En silex este grupo se reduce a 12 piezas siendo 11 de tipo «S1». Y finalmente, sólo aparecen tres en ofita siendo dos del tipo "Of1» y una del tipo «Of3».

Grupo de útiles compuestos:

Incluídas aquellas piezas retocadas que a su vez presentan una muesca, un raspador $u$ otro útil.

Se trata de un grupo muy débil y representado solamente por la cuarcita con cuatro piezas siendo tres del tipo " $\mathrm{C} 1$ " y por la ofita con una pieza del tipo "Of2".

Grupo de piezas escotadas:

En este subnivel sólo encontramos cinco muescas, fabricadas cuatro de ellas sobre cuarcita, tres del tipo «C1", y una sobre ofita del tipo «Of 1 ».

Grupo de piezas denticuladas:

Aparecen tres denticulados en cuarcita del subtipo "C1.2".

Grupo de armaduras:

Este grupo al igual que en el subnivel 18b aparece representado por una única pieza de borde rebajado fabricada en cuarcita del subtipo "C1.1".

Grupo de piezas truncadas:

Una única pieza en cuarcita del subtipo "C1.1".

Puntas:

Cuatro puntas aparecen en este subnivel; tres en cuarcita del tipo "C1" y una en silex del tipo "S1".

Limace:

Una única pieza en silex del subtipo «S1.1". 

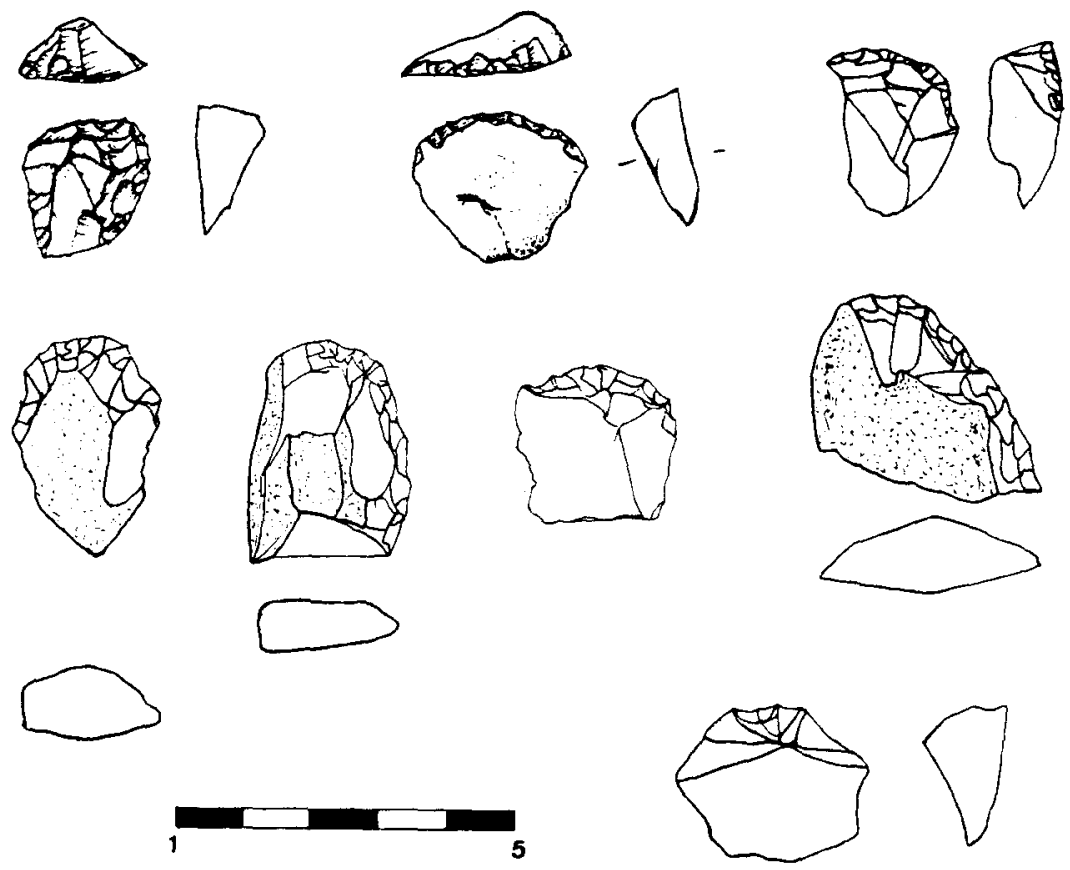

5
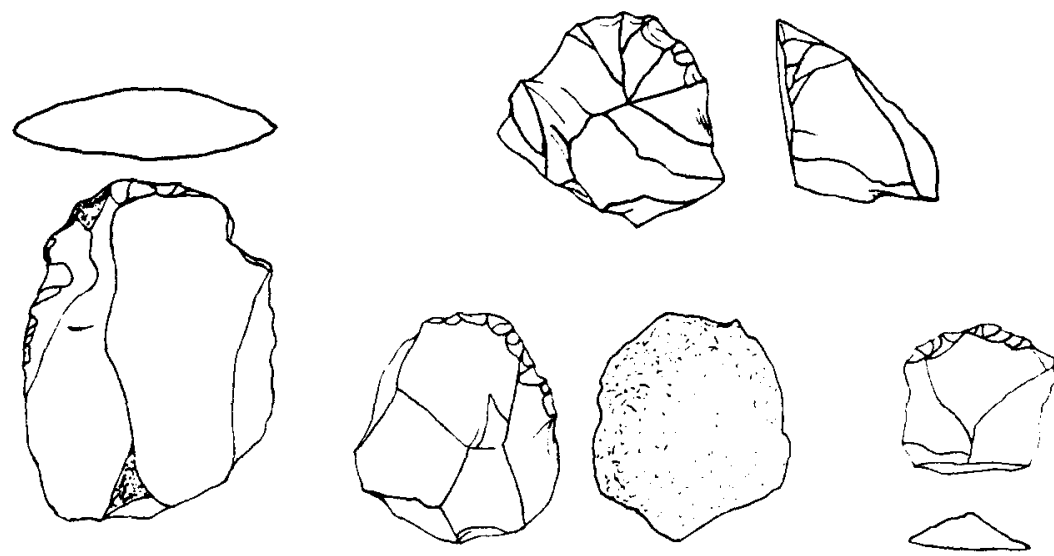

Fig. 1. Nivel 18 de la cueva del Castillo. Raspadores diversos. 

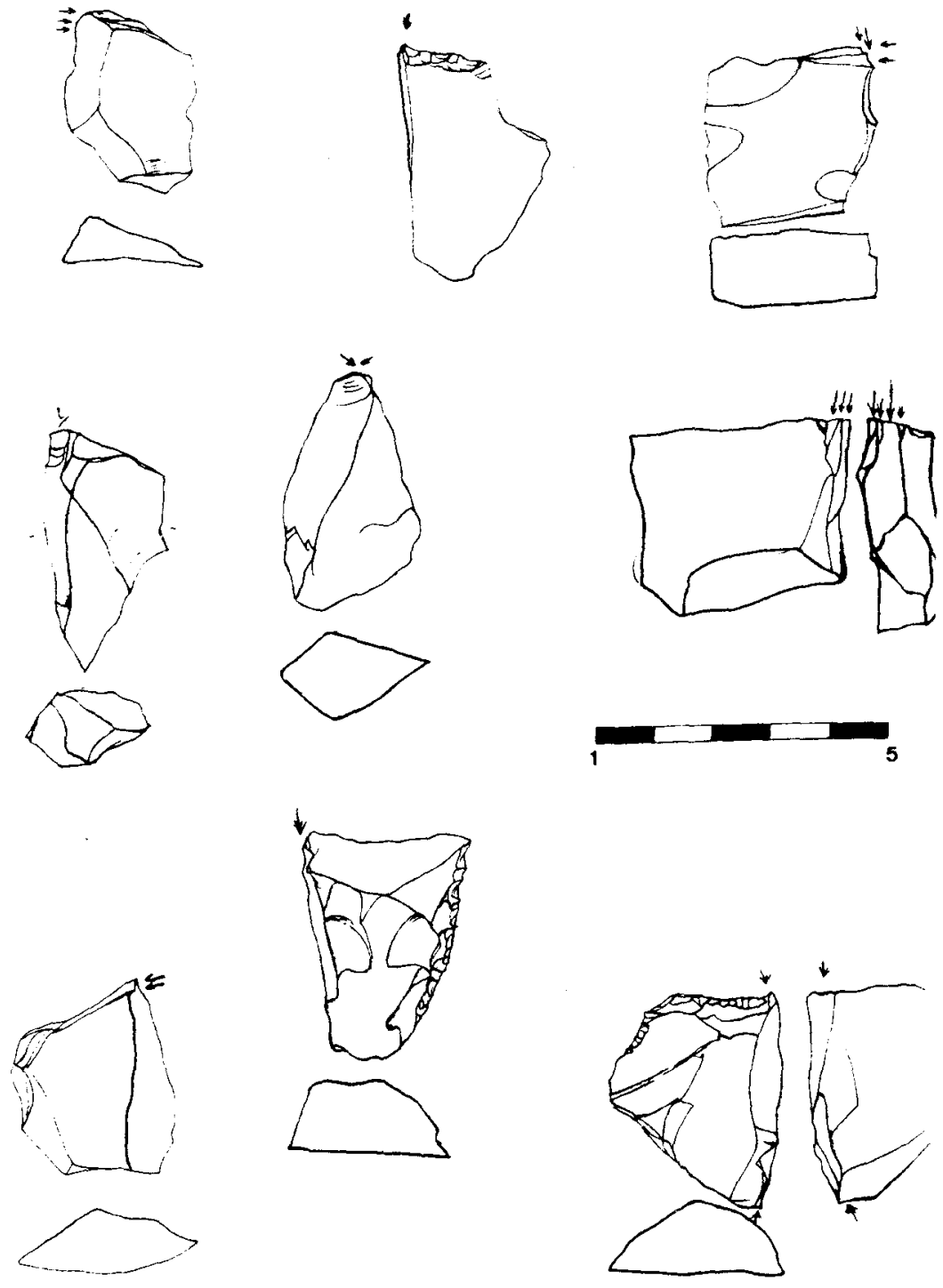

Fig. 2. Nivel 18 de la cueva de El Castillo. Buriles. 

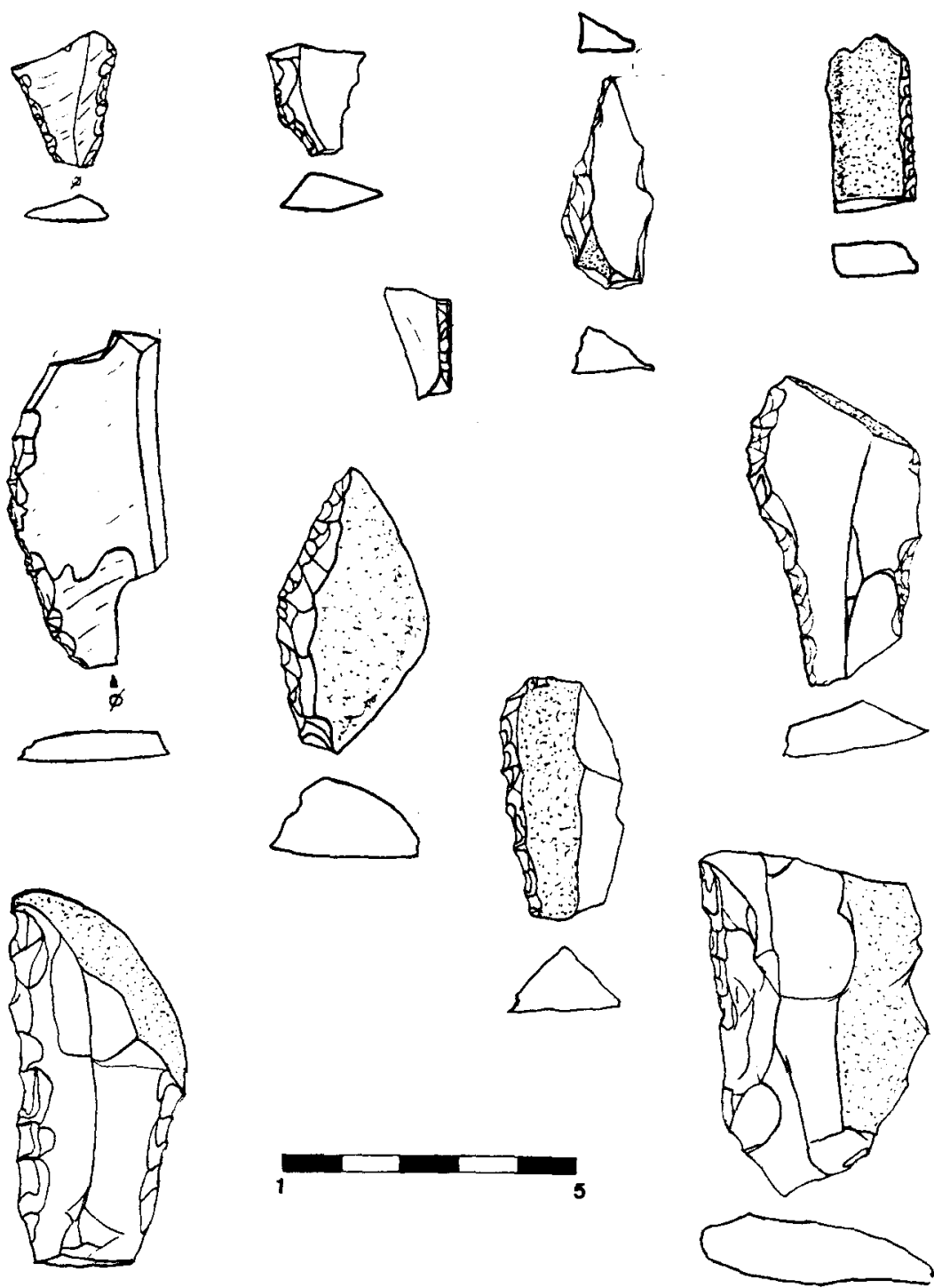

Fig. 3. Nivel 18 de la cueva de El Castillo. Útiles diversos. 
Perforador y microperforador:

Un único perforador en cuarcita del subtipo "C1.2».

Buriles:

Catorce buriles siendo 13 de cuarcita y de ellos nueve del tipo "C1", y un buril fabricado sobre silex del tipo "S3".

La industria lítica del nivel 18 se caracteriza por su elevado índice de raspadores así como de la presencia, aunque moderada, de un grupo de buriles, perforadores y hojas retocadas que nos permite relacionarlo con los inicios del Paleolítico superior y atribuirlo a un Auriñaciense arcaico (Fig. 1, 2 y 3).

La materia prima utilizada para la fabricación de los útiles sigue los mismos parámetros tanto en el subnivel $18 \mathrm{~b}$ como en el subnivel $18 \mathrm{c}$, a excepción de la caliza y el cuarzo que, aunque presentes en el conjunto de restos de talla, están ausentes en el utillaje del subnivel $18 \mathrm{c}$.

En ambos subniveles la cuarcita ha sido la materia prima más utilizada para la fabricación de los útiles seguida del silex y de una débil proporción de la ofita y de la limonita. Los útiles sobre caliza sólo están presentes en el subnivel $18 \mathrm{~b}$ y principalmente son raspadores. Por último, una pieza retocada sobre cuarzo microcristalino perteneciente también al subnivel 18b. (Fig. 4).

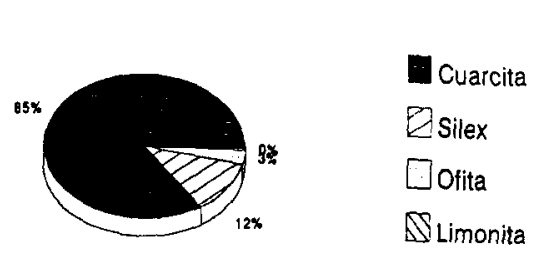

subnivel $18 \mathrm{c}$
- Cuarcita

7 silex

$1]$ Caliza

Sofita

$\square$ Limonita

Theuarzo

subnivel $18 \mathrm{~b}$

Fig. 4. Proporción de las diferentes materias primas del Auriñaciense arcaico de la cueva del "Castillo". Nota. Cifras ajustadas al porcentaje real obtenido.

El elevado índice de raspadores y la casi exclusiva utilización de la cuarcita para la fabricación de éstos junto con la manera de gestionar dicha materia a través de un desbastado recurrente centrípeto (Cabrera Valdés, et al., 1996) nos evidencia una primera intención de los hombres del auriñaciense de la cueva del Castillo: la realización de un determinado útil -el raspador carenado y en hocico- bajo la tradición de dos parámetros de la cultura musteriense que le precede, así; la continuidad en la 
selección de la primera fuente litológica de la materia prima y la tradición en la gestión tecnológica que ahora se complementa con una mayor producción y diversidad del utillaje.

A la hora de situar los niveles de la Cueva del Castillo dentro del esquema general del Auriñaciense, hemos tendido a incluirlos dentro del denominado «Auriñaciense Arcaico». Este «Auriñaciense Arcaico» fue definido por nosotros (Bernaldo de Quirós, 1982) en función de la situación estratigráfica de los niveles de las cuevas de Morín y el Pendo. Así propusimos la existencia de dos grupos (Bernaldo de Quirós, 1982: 214) uno anterior al Chatelperroniense (Pendo VIII a y VIIIb) y otro posterior (Morín 9, 8 a y 8b).

La facies tipo Morín se caracterizaría por una proporción muy alta de hojitas Dufour y un índice muy neto de raspadores auriñacienses, mientras que el índice de raederas, más bajo, indicaría una cierta distancia con el Musteriense. La facies tipo Pendo vendría caracterizada por la inexistencia de hojitas Dufour, un índice de raederas alto y una estabilidad relativa entre el índice de raspador y el de buril, aunque con predominio del primero. La presencia de raederas alcanza en los niveles del Pendo entre el 23 y el $28 \%$ y a la vez los raspadores auriñacienses aparecen en cantidades importantes (entre el 11 y el $22 \%$ ), más aun si consideramos que estos representan entre el 54 y el $62 \%$ de todos los raspadores. Como vemos estos niveles presentaban ya una curiosa mezcla de caracteres, a los que podemos unir la presencia esporádica de puntas de Chatelperron. De esta manera los niveles de Cueva Morín se situaban dentro de un clásico Auriñaciense 0 o Protoauriñaciense de la secuencia francesa (Sonneville-Bordes, 1995), mientras que los correspondientes a la Cueva del Pendo representarían una nueva facies, más cercana al Musteriense y anterior al Chatelperroniense.

Al grupo del Auriñaciense Arcaico tipo Pendo unió X. Esparza los niveles III y IVa de la Cueva de Lezetxiki (Esparza, 1985, 1993) los datos en este caso implican un predominio de las raederas, unido en el caso del nivel IIla a que los raspadores se incluyen principalmente dentro de la categoría de carenados, y destacando la presencia de núcleos prismáticos. La atribución de los autores sobre la industria del nivel Illa difiere, pues mientras que para Esparza como dijimos se incluiría dentro de un Auriñaciense Arcaico semejante al de Pendo, tenemos otro estudio de A. Baldeón $(1987,1993)$ para quien serían incluibles dentro de un Musteriense Quina, con materiales de tipo Paleolítico Superior. Esta dicotomía expresa en nuestra opinión precisamente el carácter transicional de las industrias de este tipo, situadas entre el Musteriense y el Chatelperroniense y que presentan un modelo semejante al de Castillo 18. Aunque 
geográficamente algo alejado no queremos dejar sin comentar la existencia de otros yacimientos con modelos semejantes como es el caso del Abri Olha. En los niveles musterienses $\mathrm{Fi} 4$ y Fi2 se cita "des outils épais qui rapellent plus ou moins les formes aurignaciennes, des grattoirs mouseaux, carénes, rabots, etc... qui dovient etre rangés dans les prototipes, mais ne sont pas sur lame" (Passemard, 1936), es decir de nuevo el mismo modelo que encontramos en Castillo 18.

Los niveles incluibles dentro del Auriñaciense Arcaico tipo Morin, tipo "Auriñaciense 0" o "Protoauriñaciense" se han enriquecido con los trabajos de A. Arrizabalaga sobre la Cueva de Labeko, donde se ha encontrado una interesante secuencia de Auriñaciense Arcaico tipo Morin, rico en hojitas Dufour, encima de un Chatelperroniense y con una serie de dataciones que confirma esta situación.

\section{BIBLIOGRAFÍA}

Arrizabalaga, A., 1992: "Labeko Koba (Arrasate, País Vasco): nuevos datos sobre el Paleolítico superior inicial». The Late Quaternary in the Western Pyrenean Region. Ed. Alejandro Cearreta y Félix M Ugarte. U.P.V., Gasteiz-Vitoria.

BaLdeON, A., 1987: El Musteriense del País Vasco, Vitoria, Tesis Doctoral, Universidad del País Vasco.

BALDEON, A., 1993: El yacimiento de Lezetxiki (Guipúzcoa, País Vasco). Los niveles musterienses, Munibe, 45, pp. 43-97.

Bernaldo de Quirós, F., 1982: Los inicios del Paleolítico Superior Cantábrico, Memorias del Centro de Investigaciones y Museos de Altamira, 8, Madrid.

BISChOFF, J.L., SOLER, N., MAROTO, J., et JULIA, R., 1989: Abrupt Mousterian/Aurignacian boundary at ca.40 ka bp: accelerator radiocarbon dates from l'Abreda Cave (Catalunya, Spain), Journal of Archeological Science, 16, pp. 563-576.

Bischoff, J.L., Ludwig, K., García, J.F., Carbonell, E., Vaquero, M. Stafford, T., ot Julia, A., 1994: Dating of the basal Aurignacian sandwich at Abri Romani (Catalunya, Spain) by Radiocarbon and uranium-series, Journal of Archeological Science, 21, pp. 541-551.

Cabrera Valdés, V., 1984: El yacimiento de la Cueva de "El Castillo" (Puente Viesgo, Santander), Madrid, Bibliotheca Praehistorica Hispana, XXII, Instituto Español de Prehistoria, p. $X X$.

Cabrera Valdés, V., et Bischoff, J.L., 1989: Accelerator C-14 dates for Early Upper Paleolithic (Basal Aurignacian) at el Castillo Cave (Spain), Journal of Archaelogical Science, 16, pp. 577-584.

Cabrera Valdés, V., Hoyos-Gómez, M. et Bernarldo de Quirós, F., 1993: La transición del Paleolítico Medio/Superior en la Cueva de "El Castillo": características paleoclimáticas y situación cronológica, En: El Origen del Hombre Moderno en El Suroeste de Europa, CaBRERA VALDÉS, éd., Madrid, pp. 81-101.

Cabrera Valdés, V., Hoyos Gómez, M. et Bernaldo de Quirós, F.: 1996: La transition du Paléolithique Moyen au Supérierur dans la grotte de «El Castillo»: caractéristiques paléoclimatiques et situation chronologique. En Delporte, H. et Clottes, J., Dir., Pyrénées Préhistoriques, Art et Sociétés, Editions du Comité des Travaux historiques et scientifiques, París, pp. 27-40.

Cabrera Valdés, V., Valladas, H., Bernaldo de Quirós, F. et Hoyos Gómez, M., 1996: La transition Paléolithique moyen-Paléolithique supérieur à El Castillo (Cantabrie): nouvelles datations par le carbone-14. En C.R. Acadèmie des Sciences, París, $t$. 322, série II a, p. 1093 á 1098 . 
Cabrera Valdés, V., Lloret, M., Bernaldo de Quiros, F., Hovos, M., 1996: El Auriñaciense Arcaico de la cueva del Castillo. Il Congreso de Arqueología Peninsular. Zamora, 24-27 Septiembre de 1996 (e.p.).

Deloze, V., Depaepe, P., Gouédo, V.K., Locht, J.L., 1994: Le Paléolithique moyen dans le nord du Sénonais (Yonne). dAf Documents d'Archéologie Française. Editions de la Maison des sciences de L'Homme/Paris. 1994. 276 p.

Esparza, X., 1985: El Paleolitico Superior de la Cueva de Lezetxiki (Mondragon, Guipúzcoa), Madrid, Memoria de Licenciatura, Universidad Complutense.

Esparza, X., 1993: Introducción al Paleolítico Superior de la Cueva de Lezetxiki (Mondragón, Guipúzcoa), Espacio, Tiempo y Forma, Serie l, Prehistoria y Arqueología, t. 6, pp. 31-60.

GENESTE, J.M., 1990: Développement des systèmes de production lithique au cours du Paléolithique moyen en Aquitaine septentrionale. En: Paléolithique moyen et récent et Paleolithique supérieur ancien en Europe: ruptures et transitions: examen critique des documents archéologiques. FARIZY, C., éd. Actas del coloquio internacional de Nemours, 9-10 mayo de 1988. Nemours: Apralf, p. 203-213, ill. Mémoires du Musée de Préhistoire d'lle-deFrance; 3.

Ginter, B. et Koslovsky, J., 1982: Conclusions, Excavation in the Bacho-Kiro Cave, Final Report, Polish Scientific Publishers, Varsovia, pp. 169-172.

Guilbaud, M., 1993: Debitage from the Upper Castelperronian Level at Saint-Césaire: Methodological Approach and Implications for the Transition from Middle to Upper Paleolithic. pp. 37-58. En: Contex of Late Neandertal. Implications of Multidisciplinary Research for the Transition to the Upper Paleolithic Adaptations at Saint-Césaire, CharenteMaritime, France, Levéque, F., BaCker, A.M., Gullbaud, M., éd., Monographs in World Archaelogy $n .^{\circ} 16$, Prehistory Press, p. 131.

Kunn, S.L., 1995: Mousterian Lithic Technology. An Ecological Perspective. Princenton University Press. p. 209.

LloRET, M., 1995: Análisis tecnológico de los núcleos de la cueva de La Pila (Magdaleniense Superior: nivel V y IV.4). Espacio, Tiempo y Forma, Serie I, Prehistoria y Arqueología, $t .8$., pp. 11-32.

LLORET, M., 1996: El proceso tecnológico en el Magdaleniense Superior de la cueva de La Pila (Cuchía, Cantabria). II Congreso de Arqueología Peninsular. Zamora, 24-27 Septiembre de 1996. (e.p.).

Morala, A., 1980: Observations sur le Périgordien, l'Aurignacien et leurs matières premières lithiques en Haute-Agenais. Toulouse, Ecole des Hautes Etudes en Sciences Sociales, 191 p., ill. Mémoire.

Morala, A. et TuRo, A., 1991: Relations entre matières premières lithiques et technologie: l'exemple du Paléolithique entre Dordogne et Lot. In 25 ans d'etudes technologiques en Préhistoire, bilan et perspectives. Actes de recontres 18-19-20 octobre 1990. Juan Les Pins: APDCA, pp. 159-168, ill.

PASSEMARD, E., 1936: Le Mousterien a l'Abri Olha en Pays Basque, extrait from Revue Lorraine.

Pelegrin, J., 1986: Analyse lithique: une méthode appliquée à l'étude de deux séries du Périgordien ancien - Roc de Combe couche 8 - La Côte niveau III. París: Université de Paris X.IV 584 p., fig., Tesis de Doctorado.

PHILIPPE, A., 1993; Etude technologique et typologique de l'industrie lithique du "Rocher de la Caille" (42-Loire), VI, VII. Septembre 1993. Mémoire de l'Ecole Pratique des Hautes Études. Directeur d'étude: Mr. J. Chaline.

TURQ, A., 1993: L'Approvisionnement en matières premières lithiques au Moustèrien at au début du Paléolithique supérieur dans le Nord-est du Bassin Aquitain (France)., En: El Origen del Hombre Moderno en El Suroeste de Europa, Cabrera Valoés, V., éd., Madrid, pp. 315-325.

Vitagliano, S., Piperno, M., 1990-1991: Lithic industry of level 27 Beta of the Fossellone cave (S. Felice Circeo, Latina), En: The fossil man of Monte Circeo: fifty years of studies on the Neandertals in Latium. Parco Nazionale del Circeo - October 19-21, 1989. QuaternaRIA Nova, 1, Roma, 1990-91, pp. 289-304.

Sonneville-Bordes, D., de, 1955: La Question du Perigordien II, B.S.P.F. 52, pp. 187.201. 